revista.economiaypolitica@ucuenca.edu.ec

Universidad de Cuenca

Ecuador

\title{
Análisis financiero basado en la técnica Fuzzy Logic, como instrumento para la toma de decisiones en la empresa Italimentos Cia. Ltda.
}

Saldaña Maldonado, Christian X.; Guamán, Guillermo A.

Análisis financiero basado en la técnica Fuzzy Logic, como instrumento para la toma de decisiones en la empresa Italimentos Cia. Ltda.

Revista Economía y Política, vol. XV, núm. 30, 2019

Universidad de Cuenca, Ecuador

DOI: https://doi.org/10.25097/rep.n30.2019.04

(C) Universidad de Cuenca 2019

Esta obra está bajo una Licencia Creative Commons Atribución-NoComercial-Compartirlgual 4.0 Internacional. 


\title{
Análisis financiero basado en la técnica Fuzzy Logic, como instrumento para la toma de decisiones en la empresa Italimentos Cia. Ltda.
}

\author{
Financial analysis based on the Fuzzy Logic technique, as an instrument for decision making in Italimentos Cia. \\ Ltda.
}

Christian X. Saldaña Maldonado

DOI: https://doi.org/10.25097/rep.n30.2019.04

Cooperativa de Ahorro y Crédito Jardin Azuayo Ltda., Ecuador

cristian2207@hotmail.es

Recepción: 01 Junio 2019

Guillermo A. Guamán

Aprobación: 26 Junio 2019

Universidad de Cuenca, Ecuador

guillermo.guamant@ucuenca.edu.ec

Recepción: 01 Junio 2019

Aprobación: 26 Junio 2019

\section{RESUMEN:}

Los estudios tradicionales sobre los ratios de solvencia y rentabilidad usan la matemática y los métodos tradicionales, basados en estadísticas históricas para la toma de decisiones en el corto plazo. En este sentido las decisiones de los empresarios se han basado en datos posiblemente sesgados y cerrados, sin permitir atrapar la incertidumbre que arrojan los datos al momento del análisis financiero de las empresas. En esta investigación se utiliza la técnica de análisis Fuzzy Logic que permite considerar el dato actual para la toma de decisiones correctas, atrapando la incertidumbre, a través del principio de la simultaneidad gradual que manifiesta: "Una proposición puede ser a la vez verdadera y falsa, a condición de asignar un grado a su verdad y un grado a su falsedad". La investigación es importante porque ayuda a realizar un análisis de los estados financieros acercándose a la realidad, ya que permitirá considerar dos tipos de escenarios: el mejor de los casos y el peor de los casos; así como, proporcionar estados financieros previsionales para tomar las mejores decisiones, contando con información más confiable y de calidad para la empresa. La realización de este trabajo, permitió además encontrar la diferencia entre el análisis financiero tradicional y la aplicación de la técnica Fuzzy Logic, la misma que ayudó a establecer resultados más reales para la correcta toma de decisiones en el futuro, suministrando una alternativa para la contabilidad decisional.

Palabras ClaVe: Lógica Difusa, análisis financiero, expertizaje, contraexpertizaje.

\section{Abstract:}

Traditional studies on solvency and profitability ratios use traditional mathematics and methods, based on historical statistics for short-term decision making. In this sense, the decisions of entrepreneurs have been based on possibly biased and closed data, without allowing them to trap the uncertainty that the data throw at the moment of the financial analysis of the companies. In this research, the Fuzzy Logic analysis technique is used, which allows us to consider the current data in order to make correct decisions, trapping uncertainty, through the principle of gradual simultaneity which states: "A proposition can be both true and false, provided that a degree is assigned to its truth and a degree to its falsehood". The investigation is important because it helps to make an analysis of the financial statements approaching the reality, since it will allow to consider two types of scenarios: the best of the cases and the worst of the cases; as well as, to provide provisional financial statements to take the best decisions, counting on more reliable and of quality information for the company. The accomplishment of this work, also allowed to find the difference between the traditional financial analysis and the application of the Fuzzy Logic technique, the same one that helped to establish more real results for the correct decision making in the future, providing an alternative for the decisional accounting.

KEYWORDs: Fuzzy Logic, financial analysis, expertise, contra expertise. 


\section{INTRODUCCIÓN}

En el Ecuador el sector alimenticio ha tenido un crecimiento constante debido a que las empresas buscan brindar alimentos sanos, nutritivos para los seres humanos. En base a este crecimiento, las empresas buscan ser mejores y ganar ventajas competitivas sobre otras, por lo que, los empresarios necesitan conocer su situación económica de una manera eficiente con proyección hacia el futuro.

En base a lo antes mencionado, el presente trabajo encuentra la necesidad de aplicar la lógica difusa como una técnica que ayude a conocer y aplicar números borrosos que a simple vista no se localizan en el análisis financiero. La técnica mejora las opciones de decisión y mitiga los riegos.

Diversos investigadores mencionan, que para responder a las necesidades de las empresas, se presentan dificultades al momento de procesar la información, por ejemplo: los sistemas tradicionales no cubren las necesidades informativas de los usuarios, existe imposibilidad de las personas de procesar o comprender la información financiera de la empresa, ya que la mayoría de usuarios se limitan a solo procesar la información y no a proporcionar datos para que sean analizados y ayuden en la toma de decisiones adecuadas.

También cabe mencionar, que las empresas cuentan con información de sucesos pasados, la misma, provoca que las decisiones se tomen en base a esos hechos, representando dificultades para la correcta gestión de las empresas. La aplicación de la técnica Fuzzy Logic, pretende entonces, que las empresas obtengan resultados previsionales que permitan proyecciones a futuro.

Hoy en día existen nuevos modelos económicos que han llevado a los empresarios a incorporar nuevas herramientas que les permitan analizar su situación como empresa y a partir de esta, poder compararse con el resto de organizaciones para obtener transparencias en la información presentada a los usuarios actuales y potenciales, y con ello lograr resultados de mayor satisfacción con las correctas decisiones empresariales.

La investigación presenta en primera instancia una revisión bibliográfica sobre la temática planteada, luego se determina y explica la metodología a utilizarse para el desarrollo de la propuesta, es decir, la aplicación del expertizaje y contraexpertizaje, así mismo, en los siguientes apartados se explica el proceso de esta técnica y por último se muestran los resultados obtenidos, así como las conclusiones del trabajo.

\section{REVISIÓN BIBLIOGRÁFICA}

\subsection{Conceptualizaciones básicas para el estudio}

El Análisis Financiero forma parte de un sistema o procesos de información cuya misión es la de aportar datos que permitan conocer la situación actual de la empresa y pronosticar su futuro, lo cual resulta de gran interés para gran parte de la sociedad actual, ya que los individuos son empleados por las empresas, adquieren sus bienes y servicios, invierten y obtienen información de ellas, sufren su contaminación y se benefician de los impuestos que las empresas pagan (Gil Alvarez, 2004, pág. 10).

Las nuevas técnicas han tocado cada uno de los aspectos del análisis financiero tradicional y han incorporado en él, las condiciones de incertidumbre de los problemas, así como el criterio experto. El instrumental de análisis para este nuevo enfoque se basa en la lógica difusa, los conjuntos difusos e intervalos de confianza, la matemática, el álgebra difusa y los expertones, que se han incorporado a la mayoría de modelos utilizados para el análisis financiero (Medina Hurtado, 2006, pág. 218).

Para este estudio, el análisis vertical se conceptualiza en las palabras de Lavalle Burguete (2016), que indica: "El análisis vertical toma como base el axioma matemático que enuncia que todo es igual a la suma de sus partes, en donde al todo se le asigna el valor del 100\% y a las partes un porcentaje relativo" (p.9).

El análisis horizontal, en cambio, consiste en comparar estados financieros homogéneos en periodos consecutivos. Con esto se puede observar si los resultados financieros de una empresa han sido positivos o 
negativos y también, identificar si los cambios que se han presentado merecen o no importancia para poder darles la atención pertinente. (Lavalle Burguete, 2016, pág. 11)

Por su parte, el método de análisis por razones financieras muestra un indicador más concreto de la tendencia y el comportamiento de las finanzas de la compañía objeto de estudio, ya que permite obtener indicios acerca del uso eficiente de los activos, de la rentabilidad de la empresa, de la solvencia, etc. (Lavalle Burguete, 2016, pág. 17). En este sentido, toma importancia la rentabilidad como un principio, sinónimo de ganancia, de utilidad, de beneficio, de lucro. Presupone la realización de negocios con márgenes positivos e implica, en el largo plazo, que el dinero que ingresa a la empresa sea mayor que el dinero que sale de la misma. (Faga y Ramos Mejía, 2006, pág. 14)

En suma a la terminología requerida para la investigación, un ratio es el cociente entre magnitudes que tienen una cierta relación de tipo económico y que por este motivo se comparan. Es decir, el resultado de este cociente nos proporcionará una información relevante sobre algún aspecto económico de la empresa. Las principales utilidades de los ratios están en relación a qué, con quién o cuando los comparamos o interrelacionamos. (García Parra y Jordà Lloret, 2004, pág. 150)

La técnica base de este estudio se conceptualiza en las palabras de Lotfy A. Zadeh (como se citó en Tinto, Molina, Chávez y Mosquera, 2016), en donde se describe que la Lógica Difusa fue introducida por primera vez con ese nombre en 1965. La creciente disciplina permite trasladar el lenguaje común; subjetivo, impreciso y en muchos casos no cuantificable, a un lenguaje matemático formal. A diferencia de la lógica clásica, los estudios a partir de la lógica difusa tienen la capacidad de reflejar los modos más usuales de razonamiento a partir de considerar que la certeza de una proposición es una cuestión de grados. (pág. 7)

Trillas y Gutiérrez (1992) afirman que al hablar de lógica difusa se inicia explicando las variables denominadas lingüísticas que permiten analizar perspectivas numéricas de cero a uno con intervalos de medición, por lo que, la lógica difusa es un tipo de lógica multivaluada y se caracteriza por querer cuantificar esta incertidumbre. La lógica borrosa o difusa se basa en el principio de "Todo es cuestión de grado".

La lógica difusa es conceptualmente fácil de entender porque los conceptos matemáticos que maneja son simples, además, es flexible, ya que se puede incrementar su complejidad sin necesidad de re elaborar el análisis desde su inicio y es tolerante a los datos imprecisos: los incorpora dentro de su filosofía. (D 'Negri y De Vito, 2006, págs. 129-133)

Por último, es necesario anotar que la aplicación de esta técnica tiene como principal dificultad la clasificación de la variales y la interpretación de los datos difusos.

Para este estudio, se tornan importantes también las reflexiones y comentarios en el ámbito de aplicación de la Contabilidad, las mismas que explican muchos problemas que se revelan ambiguos e imprecisos, por ejemplo: el ajuste de los estados contables a la situación financiera real de la empresa, investigar el significado de las desviaciones en un sistema de control interno débil, incrementar el tamaño de la muestra si la materialidad es débil en el control interno, localizar los costes indirectos utilizando bases adecuadas y considerar varios productos como uno solo cuando sus niveles de ventas son pequeños. (Reig Mullor, Sansalvador Selles, y Trigueros Pina, 2000, pág. 93)

En cuanto a la Teoría del expertizaje y contraexpertizaje, en primera instancia es necesario indicar que se trata de una de las herramientas esenciales utilizadas en lógica difusa para disminuir la entropía y afinar los valores analizados; es la escala semántica endecadaria, la cual se adapta a once expresiones lingüísticas y por tanto subjetivas e inciertas, pero con un nivel sensato de presunción $\alpha$ de verdad comprendido en el intervalo $[0 ; 1]$, donde el cero representa la inequívoca no pertenencia y el uno la absoluta pertenencia.

En este orden de ideas, se define el expertizaje como el proceso de consulta a grupos de expertos en relación a un tema determinado, con el fin de acotar la incertidumbre. Siguiendo la teoría del expertizaje, se puede consultar a expertos en activos intangibles, quienes darán respuestas puntuales a partir de las cuales se determinará un intervalo de confianza que servirá de inicio a tal proceso. (Rico F., 2010, pág. 129) 
En referencia al concepto valuación, se entiende como las referencias a un dato numérico en una escala adecuada de valores con la cual se pondera un fenómeno percibido por nuestros sentidos o experiencia. Esta valuación se puede expresar en cualquier lenguaje humano. Para las valuaciones en la matemática borrosa, se emplean ponderaciones entre 0 (falso) y 1 (verdadero), sin confundir los términos de valuación y probabilidad, ya que la valuación es un dato subjetivo suministrado por una o varias personas y una probabilidad es un dato objetivo probado a través del tiempo y aceptado de forma general.

De esta manera, las valuaciones poseen infinitas formas o escalas según las cuales se presentan, la más difundida es la escala endecadaria que agrupa once alternativas expresadas en la tabla 1.

TABLA 1.

Escala Endecadaria

\begin{tabular}{ll}
\hline $\begin{array}{l}\text { Grado de } \\
\text { Presunción }\end{array}$ & Incidencia \\
\hline 0 & FALSO \\
0,1 & Prácticamente \\
0,2 & falso \\
0,3 & Casi falso \\
0,4 & Bastante falso \\
& Más falso que \\
0,5 & verdadero \\
& Tan falso \\
0,6 & como \\
& verdadero \\
0,7 & Más verdadero \\
0,8 & Bae falso \\
0,9 & verdadero \\
1 & Casi \\
& Verdadero \\
& Prácticamente \\
& verdadero \\
& VERDADERO \\
\hline
\end{tabular}

Tinto, 2015

En este punto, la noción de intervalo de confianza debe ser analizado, ya que para expresar las valuaciones se necesita un rango en el que el intervalo de confianza será el número más sencillo, ya que sólo posee dos valores, en donde $\mathrm{A}$ y $\mathrm{B}$ deben ser siempre inferiores o iguales a uno. Así, A ni B no pueden ser mayores a uno y además, $A$ nunca puede ser mayor que $B$, sólo podría llegar a igualarlo $(0 \leq \mathrm{A} \leq \mathrm{B} \leq 1)$. (Tinto Arandes, 2015, pág. 6)

\subsection{Marco referencial}

Tinto, Molina, Chavez y Mosquera (2016) en su artículo “Automatización fuzzy aplicado en la Contabilidad Decisional", analizan:

Un modelo automatizado que permita la evaluación de las cifras contables para la correcta toma de decisiones a mediano y largo plazo, partiendo de los estados financieros (...). Con el modelo se analiza la solvencia financiera de la empresa y el estudio del beneficio a través de los ratios en la incertidumbre para la toma de decisiones acertadas que debe poseer el empresario actual.

Para el diseño del modelo, los investigadores recurren a realizar entrevistas a un conjunto de expertos de las empresas bananeras de la Provincia de El Oro - Ecuador, para determinar la opinión agregada de ellos y por medio de las técnicas de expertizaje y contraexpertizaje, obtener el dato contable en forma de intervalo de 
confianza. Se emplean valores entre 0 y para trabajar con dicho número borroso y de esta forma se construyen las preguntas utilizadas en la investigación, para cada una de las partidas que requieran análisis, obteniendo intervalos de confianza que reflejan la situación desde un extremo pesimista a un extremo optimista. (Tinto, Molina, Chavez, \& Mosquera, 2016)

Dicho trabajo comprende la automatización de los cálculos independientemente del número de expertos que intervengan en el proceso y de esta forma partiendo de los últimos estados de situación de la empresa se construyen los estados previsionales y se obtienen los ratios para una correcta toma de decisiones.

Luego de la aplicación del método se pasa a un conocimiento superior, donde los resultados no pasan a ser datos estadísticos, sino sirven para calcular los ratios de la empresa y apoyar la definición de decisiones que ayuden a tomar las previsiones adecuadas.

Tinto (2015) analiza la implementación de tecnología de avanzada (fuzzy-set) aplicada a los sistemas de control y gestión en las cadenas agroalimentarias del Ecuador, en donde el objetivo general del proyecto es:

Generar una nueva línea de investigación, promotora del conocimiento, para la creación de sistemas de automatización y robótica en la industria alimentaria del Ecuador”, esta permitirá controlar los factores que afectan a las cadenas agroalimentarias y agroindustriales, aplicando tecnología fuzzy, para la eficiente gestión, control y gerencia en los procesos productivos, con criterios de sustentabilidad, responsabilidad social, y con clara orientación a la solución de los problemas actuales $(\mathrm{s} / \mathrm{n})$.

La metodología del trabajo se centra en el empleo de la lógica difusa a través de la técnica del expertizaje y el contraexpertizaje. Todas estas herramientas permitirán globalizar las incidencias directas e indirectas existentes entre un número de causas y un grupo de efectos que pueden condicionar, en ciertos momentos, la estructura financiera y económica de una empresa o sector. (Tinto Arandes, 2015)

En este punto, se definen dos conceptos de la lógica difusa: la valuación y los intervalos de confianza, ya que gran parte de los procesos difusos se basan en ellos.

Para los autores Luna, Tinto, Sarmiento y Cisneros (2018) en su artículo "Estudio de rentabilidad para el lanzamiento de un nuevo producto aplicando el enfoque difuso", analizan "la determinación del precio de venta y la utilidad bajo el enfoque difuso, para demostrar la factibilidad de comercializar en el mercado una bota de vestir para dama, que contará con el aporte del conocimiento de los artesanos expertos en calzado del cantón Gualaceo."

En este artículo se desarrollan herramientas de avanzada como:

1. Alfa cortes, que consiste en trabajar a ciertos niveles de posibilidad de que ocurra el suceso.

2. Análisis en la incertidumbre con base en los números borrosos triangulares en forma de intervalos.

3. Análisis de costos, precio y utilidad bajo el enfoque difuso.

La metodología utiliza números borrosos triangulares, que es aquel subconjunto borroso que se forma por una secuencia finita e infinita de intervalos de confianza, que surgen de asignar un nivel de confianza a los valores de un conjunto referencial. Esta aplicación se verá reflejada de la información obtenida de los artesanos de calzado del cantón Gualaceo; los datos hacen relación a los costos totales y la producción realizada en un mes.

"La utilización de los números borrosos triangulares y la aplicación de los alfa cortes, permite a los artesanos de calzado, ofertar su producto dentro de una banda de precios, que facilita un mejor manejo financiero para lograr la mejor utilidad posible”. (Tinto, Luna, \& Cisneros, 2017). Generando un aporte cognitivo a los artesanos de calzado considerados en la investigación, con el fin de implementar "este método para la determinación del precio de venta y la utilidad que se desearía obtener entre un numero borroso triangular, con el propósito de que el fabricante puede ofertar su producto dentro de un intervalo, teniendo un precio mínimo y máximo de venta y cuya utilidad está representada de la misma manera" (Tinto, Luna, \& Cisneros, 2017).

Los autores Díaz, Coba, Moreno y Santamaría (2017) analizan la Lógica Difusa Aplicada a los Ratios Financieros en el Sector Cooperativo del Ecuador, en donde: 
Al hablar de lógica difusa se inicia explicando las variables denominadas lingüísticas que permiten analizar perspectivas numéricas de cero (0) a uno (1). Estas variables lingüísticas permiten al experto establecer una valoración cualitativa de datos numéricos que pueden medir el comportamiento difuso de cualquier área de conocimiento (p. 65)

En el mismo estudio, se menciona que "al generar el análisis financiero tradicional a través de ratios puede omitirse información relevante al momento de tomar una decisión. En este sentido la lógica difusa puede constituirse en una herramienta que permita dar una mejor perspectiva y para realizar un análisis más profundo de la situación financiera de una organización" (Díaz, Coba, Moreno, \& Santamaría, 2017).

Por último, Díaz, Coba, Moreno y Santamaría (2017), explican que la metodología difusa suele utilizarse, de manera general, en casos que presentan complejidad muy alta en el proceso de análisis y por esta situación, es improbable precisar con modelos matemáticos tradicionales, dando paso de esta forma, "a conceptos subjetivos para la precisión del análisis, donde, se puede establecer variables que ayudan a la comprensión total y simplificada de un hecho" (p.66).

\section{Metodología}

La presente investigación es exploratoria y descriptiva ya que permitirá concluir y realizar una comparación entre el análisis financiero tradicional y la aplicación de la herramienta Fuzzy Logic, añadiendo también una comparación con otras investigaciones.

El tipo de muestreo a utilizarse es no probabilístico, recurriendo al método de conveniencia para la obtención de los datos, en donde se realizó entrevistas a expertos que trabajan en la empresa.

En primer lugar, se realiza un análisis financiero tradicional, para lo cual se utilizan los Estados Financieros de la empresa ITALIMENTOS CIA. LTDA. En este punto se analizará de forma vertical y horizontal los datos proporcionados por la misma, luego se calculan las razones financieras para la misma.

Scott y Brigham (2013), expresan las siguientes razones financieras:
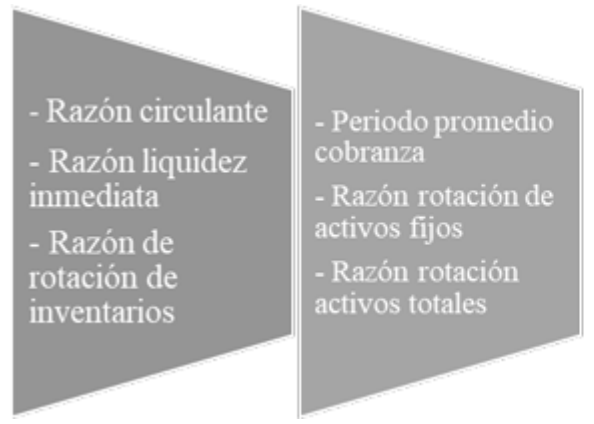

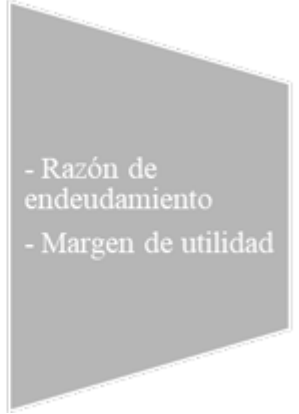

GRÁFICO 1.

\section{Razones Financieras}

Scott y Brigham, 2013

En segundo lugar, para el desarrollo del modelo, se realizan entrevistas a un grupo de expertos de la empresa ITALIMENTOS CIA. LTDA., dedicada a la industria alimenticia. Con esto se espera obtener una opinión profesional y de esta manera poder aplicar la técnica de expertizaje y contraexpertizaje, obteniendo un dato contable en forma de intervalo de confianza.

Para trabajar con estos números borrosos se establecen valores entre cero y uno, según la escala endecadaria presentada anteriormente. Luego se detalla el proceso a seguir al aplicar el Expertizaje, donde:

- Se vacía la información de los expertos en la escala endecadaria.

- Se normaliza la serie, dividiendo los valores para el número de expertos. 
- Se acumula la frecuencia relativa, con lo que se obtiene el expertón, que consiste en sumar los valores de abajo hacia arriba hasta totalizar la unidad. El valor resultante de la suma se divide entre 10 que representa el número de cifras que componen el grado de presunción desde 0,1 hasta 1 ,

Con el dato obtenido se debe aplicar el contraexpertizaje, definido en palabras de Rico F. (2010) como un procedimiento aritmético con base en los subconjuntos borrosos que permite disminuir la entropía en las variables o categorías estudiadas mediante la aplicación de la siguiente fórmula:

$$
\mathrm{Ei}+[E s-E i] x \text { experton }
$$

Donde:

$\mathrm{Ei}=$ Límite inferior

Es $=$ Límite superior

Experton $=$ Resultado del expertizaje

Al finalizar la aplicación de los dos tipos de análisis, se realiza una comparación entre el análisis tradicional y el modelo difuso, luego de ello, se observa como es la volatilidad de los resultados obtenidos y así se concluye con información eficiente para la toma de decisiones de la empresa.

\section{Desarrollo y Aplicación}

\subsection{Análisis financiero}

En este punto se analiza la información contable de la empresa, con el fin de observar cual es la situación actual de la misma y utilizar esta como base para la aplicación del modelo difuso. 


\subsubsection{Estado de situación financiera}

TABLA 2.

Análisis Financiero Estático

\begin{tabular}{|c|c|c|c|c|c|c|}
\hline $\begin{array}{l}\text { Estado de } \\
\text { Situación } \\
\text { Financiera }\end{array}$ & 2015 & & 2016 & & 2017 & \\
\hline $\begin{array}{l}\text { Activos } \\
\text { Corrientes }\end{array}$ & 11.299 .980 & $54,32 \%$ & 12.000 .854 & $51,33 \%$ & 13.751 .838 & $54,33 \%$ \\
\hline $\begin{array}{l}\text { Activos No } \\
\text { Corrientes }\end{array}$ & 9.504 .160 & $45,68 \%$ & 11.379 .653 & $48,67 \%$ & 11.560 .471 & $45,67 \%$ \\
\hline $\begin{array}{l}\text { Total } \\
\text { Activos }\end{array}$ & 20.804 .139 & $100,00 \%$ & 23.380 .507 & $100,00 \%$ & 25.312 .310 & $100,00 \%$ \\
\hline $\begin{array}{l}\text { Pasivos } \\
\text { Corrientes }\end{array}$ & 8.405 .004 & $40,40 \%$ & 9.261 .863 & $39,61 \%$ & 10.352 .966 & $40,90 \%$ \\
\hline $\begin{array}{l}\text { Pasivos No } \\
\text { Corrientes }\end{array}$ & 3.930 .013 & $18,89 \%$ & 4.261 .855 & $18,23 \%$ & 3.438 .003 & $13,58 \%$ \\
\hline $\begin{array}{l}\text { Total } \\
\text { Pasivos }\end{array}$ & 12.335 .017 & & 13.523 .719 & & 13.790 .969 & \\
\hline $\begin{array}{l}\text { Capital y } \\
\text { Aportes }\end{array}$ & 2.921 .150 & $14,04 \%$ & 3.421 .150 & $14,63 \%$ & 3.921 .150 & $15,49 \%$ \\
\hline $\begin{array}{l}\text { Reservas + } \\
\text { ORI + } \\
\text { Adop. NIIF }\end{array}$ & 942.641 & $4,53 \%$ & 724.621 & $3,10 \%$ & 1.104 .291 & $4,36 \%$ \\
\hline $\begin{array}{l}\text { Resultados } \\
\text { acumulados }\end{array}$ & 3.530 .512 & $16,97 \%$ & 3.701 .590 & $15,83 \%$ & 4.654 .061 & $18,39 \%$ \\
\hline $\begin{array}{l}\text { Resultado } \\
\text { del ejercicio }\end{array}$ & 1.074 .819 & $5,17 \%$ & 2.009 .428 & $8,59 \%$ & 1.841 .839 & $7,28 \%$ \\
\hline $\begin{array}{l}\text { Total } \\
\text { Patrimonio } \\
\text { Total }\end{array}$ & 8.469 .122 & & 9.856 .789 & & 11.521 .341 & \\
\hline $\begin{array}{l}\text { Pasivos + } \\
\text { Patrimonio }\end{array}$ & 20.804 .139 & $100,00 \%$ & 23.380 .508 & $100,00 \%$ & 25.312 .310 & $100,00 \%$ \\
\hline
\end{tabular}

Italimentos Cía. Ltda.

Se observa, en la tabla 2, que en la estructura económica de la empresa para el año 2017, los activos corrientes representan un 54,33\%, en relación al 100\% del activo total, mientras que sus activos no corrientes representan el 45,67\%, lo que significa que los activos a corto plazo han incrementado. De igual manera se puede observar la misma tendencia en los años 2015 y 2016.

Para el año 2017, los pasivos corrientes representan un 40,90\%, lo que significa que la empresa es financiada por recursos ajenos, donde implica que el incremento en los activos circulantes le sirve para cubrir parte de sus deudas a corto plazo. 
TABLA 3.

Análisis financiero dinámico

\begin{tabular}{|c|c|c|c|c|c|}
\hline $\begin{array}{l}\text { Estado de } \\
\text { Situación } \\
\text { Financiera }\end{array}$ & 2015 & 2016 & & 2017 & \\
\hline $\begin{array}{l}\text { Activos } \\
\text { Corrientes }\end{array}$ & 11.299 .980 & 12.000 .854 & $6,20 \%$ & 13.751 .838 & $14,59 \%$ \\
\hline $\begin{array}{l}\text { Activos No } \\
\text { Corrientes }\end{array}$ & 9.504 .160 & 11.379 .653 & $19,73 \%$ & 11.560 .471 & $1,59 \%$ \\
\hline $\begin{array}{l}\text { Total } \\
\text { Activos }\end{array}$ & 20.804 .139 & 23.380 .507 & & 25.312 .310 & \\
\hline $\begin{array}{l}\text { Pasivos } \\
\text { Corrientes }\end{array}$ & 8.405 .004 & 9.261 .863 & $10,19 \%$ & 10.352 .966 & $11,78 \%$ \\
\hline $\begin{array}{l}\text { Pasivos No } \\
\text { Corrientes }\end{array}$ & 3.930 .013 & 4.261 .855 & $8,44 \%$ & 3.438 .003 & $-19,33 \%$ \\
\hline $\begin{array}{l}\text { Total } \\
\text { Pasivos }\end{array}$ & 12.335 .017 & 13.523 .719 & & 13.790 .969 & \\
\hline $\begin{array}{l}\text { Capital y } \\
\text { Aportes }\end{array}$ & 2.921 .150 & 3.421 .150 & $17,12 \%$ & 3.921 .150 & $14,61 \%$ \\
\hline $\begin{array}{l}\text { Reservas + } \\
\text { ORI }+ \\
\text { Adop. NIIF }\end{array}$ & 942.641 & 724.621 & $-23,13 \%$ & 1.104 .291 & $52,40 \%$ \\
\hline $\begin{array}{l}\text { Resultados } \\
\text { acumulados }\end{array}$ & 3.530 .512 & 3.701 .590 & $4,85 \%$ & 4.654 .061 & $25,73 \%$ \\
\hline $\begin{array}{l}\text { Resultado } \\
\text { del ejercicio }\end{array}$ & 1.074 .819 & 2.009 .428 & $86,96 \%$ & 1.841 .839 & $-8,34 \%$ \\
\hline $\begin{array}{l}\text { Total } \\
\text { Patrimonio }\end{array}$ & 8.469 .122 & 9.856 .789 & & 11.521 .341 & \\
\hline
\end{tabular}

Italimentos Cía. Ltda

En la tabla 3 se observa que en el año 2016, el activo corriente ha tenido un incremento del $6.20 \%$ con relación al año anterior, mientras que para el año 2017 incrementó en $14.59 \%$, este crecimiento se debe a que existe más inventario en la empresa. En cuanto al activo no corriente, para el año 2016, se incrementó en $19.73 \%$ con relación al año anterior y para el 2017 se tuvo un incremento mínimo de $1.59 \%$, demostrando que la empresa tuvo fuertes inversiones, generando una imagen empresarial sólida.

Los pasivos corrientes para el 2016 incrementaron un 10.19\% con relación al 2015, mientras que para el 2017 tuvieron un incremento de $11.78 \%$, lo que nos dice que la empresa trabaja con recursos ajenos para hacer frente a sus actividades diarias. Los pasivos no corrientes para el año 2016 incrementaron en un $8.44 \%$ y para el 2017 disminuyeron considerablemente en $-19.33 \%$ dando a conocer que la empresa no cuenta con deudas a largo plazo.

En lo referente al patrimonio de la empresa para el año 2016 tiene un crecimiento del $16 \%$ y para el año 2017 del 17\%, esto se debe a que la empresa está incrementando su utilidad y a su vez el capital, demostrando el crecimiento que tiene la empresa de un año a otro. 


\subsubsection{Estado de pérdidas y ganancias}

TABLA 4.

Análisis financiero estático

\begin{tabular}{|c|c|c|c|c|c|c|}
\hline $\begin{array}{l}\text { Estado de } \\
\text { Resultados }\end{array}$ & 2015 & & 2016 & & 2017 & \\
\hline $\begin{array}{l}\text { Total } \\
\text { Ingresos }\end{array}$ & 40.281 .810 & $100,00 \%$ & 40.390 .464 & $100,00 \%$ & 47.622 .398 & $100,00 \%$ \\
\hline $\begin{array}{l}\text { Total } \\
\text { Costos }\end{array}$ & 29.769 .893 & $73,90 \%$ & 28.542 .864 & $70,67 \%$ & 34.384 .648 & $72,20 \%$ \\
\hline $\begin{array}{l}\text { Total } \\
\text { Gastos }\end{array}$ & 8.789 .455 & $21,82 \%$ & 8.643 .434 & $21,40 \%$ & 10.161 .957 & $21,34 \%$ \\
\hline $\begin{array}{l}\text { 15\% Part. } \\
\text { Laboral }\end{array}$ & 258.369 & $0,64 \%$ & 480.625 & $1,19 \%$ & 461.369 & $0,97 \%$ \\
\hline $\begin{array}{l}\text { Impuesto } \\
\text { Causado }\end{array}$ & 389.273 & $0,97 \%$ & 714.114 & $1,77 \%$ & 772.584 & $1,62 \%$ \\
\hline $\begin{array}{l}\text { Utilidad } \\
\text { Neta }\end{array}$ & 1.074 .819 & $2,67 \%$ & 2.009 .428 & $4,98 \%$ & 1.841 .839 & $3,87 \%$ \\
\hline
\end{tabular}

Italimentos Cía. Ltda.

Para el análisis (tabla 4) se ha asignado el 100\% al total de ingresos, donde se observa que el costo de ventas es el rubro más representativo durante todos los años. Se puede notar también que los gastos generales representan un porcentaje considerable con respecto a los ingresos en todos los años de estudio, a pesar de esto, la utilidad neta que se obtiene es poco significativa, debido a un alto costo de ventas, sin embargo, la empresa continúa su crecimiento y demuestra una buena utilización de sus recursos para generar resultados positivos.

TABLA 5.

Análisis financiero dinámico

\begin{tabular}{|c|c|c|c|c|c|}
\hline $\begin{array}{l}\text { Estado de } \\
\text { Resultados }\end{array}$ & 2015 & 2016 & & 2017 & \\
\hline $\begin{array}{l}\text { Total } \\
\text { Ingresos }\end{array}$ & 40.281 .810 & 40.390 .464 & $0,27 \%$ & 47.622 .398 & $17,91 \%$ \\
\hline $\begin{array}{l}\text { Total } \\
\text { Costos }\end{array}$ & 29.769 .893 & 28.542 .864 & $-4,12 \%$ & 34.384 .648 & $20,47 \%$ \\
\hline $\begin{array}{l}\text { Total } \\
\text { Gastos }\end{array}$ & 8.789 .455 & 8.643 .434 & $-1,66 \%$ & 10.161 .957 & $17,57 \%$ \\
\hline $\begin{array}{l}\text { 15\% Part. } \\
\text { Laboral }\end{array}$ & 258.369 & 480.625 & $86,02 \%$ & 461.369 & $-4,01 \%$ \\
\hline $\begin{array}{l}\text { Impuesto } \\
\text { Causado }\end{array}$ & 389.273 & 714.114 & $83,45 \%$ & 772.584 & $8,19 \%$ \\
\hline $\begin{array}{l}\text { Utilidad } \\
\text { Neta }\end{array}$ & 1.074 .819 & 2.009 .428 & $86,96 \%$ & 1.841 .839 & $-8,34 \%$ \\
\hline
\end{tabular}

Italimentos Cía. Ltda.

Del total de ingresos del año 2016 no se ha tenido un crecimiento significativo ya que fue de $0.27 \%$ en comparación al año 2015, mientras que para el año 2017 la empresa tiene un crecimiento en sus ventas del $17.91 \%$, lo que indica que la empresa se encuentra en crecimiento. Esto se puede verificar en la tabla 5.

Asimismo, sus costos para el año 2016 no tuvieron incremento, mientras que para el 2017 crecieron en $20.47 \%$, esto se debe a la relación directa que este rubro tiene con las ventas. De igual manera se observa un aumento en los gastos que la empresa posee para el año 2017. 
En cuanto al margen de utilidad de la empresa, para el año 2016 tuvo un crecimiento considerable, llegando a un $86.96 \%$, mientras que para el 2017 disminuyó un $-8.34 \%$ debido a que también incrementaron los costos y el gasto anual.

\subsection{Razones financieras}

TABLA 6.

Razones Financieras

\begin{tabular}{lll}
\hline Indicador & Empresa & Industria \\
\hline $\begin{array}{l}\text { Razón } \\
\text { circulante }\end{array}$ & $=1,33$ & 2.44 \\
$\begin{array}{l}\text { Razón liquidez } \\
\text { inmediata }\end{array}$ & $=0,74$ & 1.74 \\
$\begin{array}{l}\text { Razón de } \\
\text { rotación de } \\
\text { inventarios }\end{array}$ & $=5,68$ & 6.57 \\
$\begin{array}{l}\text { Periodo } \\
\text { promedio }\end{array}$ & $=48,02$ & 52.82 \\
$\begin{array}{l}\text { cobranza } \\
\text { Razón rotación } \\
\text { de activos }\end{array}$ & $=4,28$ & 4.38 \\
$\begin{array}{l}\text { fijos } \\
\text { Razón rotación } \\
\text { activos totales }\end{array}$ & $=1,86$ & 1.66 \\
$\begin{array}{l}\text { Razón de } \\
\text { endeudamiento }\end{array}$ & $=0,54$ & 0.47 \\
$\begin{array}{l}\text { Margen de } \\
\text { utilidad }\end{array}$ & $=0,04$ & 0.05 \\
$\begin{array}{l}\text { Rendimiento } \\
\text { sobre el capital } \\
\text { Contable }\end{array}$ & $=0,47$ & 0.40 \\
$\begin{array}{l}\text { ROA A } \\
\text { ROE }\end{array}$ & $=0,07$ & 0.07 \\
\hline
\end{tabular}

Empresa y Sector Alimenticio

\section{Razón circulante:}

Refleja que, por cada dólar de obligaciones a corto plazo, cuenta con $\$ 1,33$ de activo circulante para cubrir dichas obligaciones. En comparación con el sector industrial, el activo circulante se encuentra por debajo, lo que refleja que la empresa analizada tiene liquidez para cubrir sus deudas, pero puede mejorar según la naturaleza de la misma.

\section{Razón de liquidez inmediata:}

También llamada prueba acida, debido a que se resta el valor de inventarios porque se trata de un valor que demora más tiempo en convertirse en dinero. Reflejando que, por cada dólar de obligaciones a corto plazo, la empresa cuenta con $\$ 0.74$ para hacer frente a dichas obligaciones. Con relación a la industria está por debajo por lo que la empresa cuenta con mucho inventario, afectando a su liquidez.

Razón de rotación de inventarios:

Indica que los inventarios han rotado 5.68 veces en el año, situación positiva ya que indica que la empresa aumenta sus ventas. Se puede notar que la empresa rota menos veces el inventario en comparación con lo de la industria que es de 6.57 veces, pero no por eso la empresa está mal, lo que se recomienda es acercase a lo que dice el sector.

Periodo promedio de cobranza: 
Con este indicador se aprecia que la empresa recupera su cartera cada 48 días. Situación positiva que permite nuevas inversiones y además, se podría decir que cuenta con una buena política de recuperación de cartera. Al comparar con el sector industrial, se observa que la empresa está por debajo, indicador beneficioso para futuras inversiones.

Razón de rotación de activos fijos:

Durante el año los activos fijos han rotado 4 veces, lo que significa que el dinero invertido proporciona una mayor productividad de los activos fijos y por consiguiente, una empresa más eficiente. Al comparar a la empresa con la situación del sector industrial que se encuentra en el mismo nivel, se verifica un buen manejo de sus activos.

Razón de rotación de activos totales:

Indica que todo está en movimiento y que la empresa utiliza sus activos para generar beneficios futuros, indicando que roto 1.86 veces en el año, con relación a la industria, la empresa se encuentra por encima, lo que es bueno ya que a mayor rotación mejor es el uso de sus activos para incrementar sus ingresos.

Razón de endeudamiento:

Indica que por cada dólar que se tiene en activos, se debe que pagar $\$ 0,54$, es decir, es ajeno, significando un alto nivel de endeudamiento considerado necesario para sus actividades diarias.

\section{Margen de utilidad:}

Explica que por cada dólar que se vende, la empresa gana $\$ 0,04$, lo cual refleja una buena situación financiera debido al incremento que ha tenido en sus ingresos. Al comparar con la industria se observa que está por debajo, pero para la empresa es bueno, ya que su actividad genera ganancias.

Rendimiento sobre capital contable:

Demuestra que cada dólar que un accionista tiene en la empresa, le genera $\$ 0,47$ de utilidad; es decir, el rendimiento que se obtiene sobre el capital es alto lo cual es beneficioso para los socios de la empresa.

Roa:

Este indicador muestra como cada dólar que la empresa posee en activos, le genera un retorno del 7\%. Es decir, la empresa aprovecha sus activos para generar beneficios.

Roe:

Este indicador muestra que el $15.98 \%$ tienen de retorno por la inversión que realizan los accionistas en la empresa.

\section{Aplicación de la PROPUEsta}

Para el diseño del modelo se realizan entrevistas a un conjunto de expertos de la empresa, con el objetivo de obtener una opinión y aplicar la técnica del expertizaje y contraexpertizaje, y así obtener el dato contable. Para el desarrollo de modelo difuso se realizan preguntas de la siguiente manera:

¿Considera usted que, de acuerdo a las situaciones económicas actuales, para el siguiente año aumenten en un $15 \%$ las ventas de la empresa Italimentos Cía. Ltda.? Escoja entre las siguientes posibilidades:

0 Las ventas no alcanzarán un aumento del $15 \%$.

0.1 Las ventas prácticamente no alcanzarán un aumento del 15\%.

0.2 Las ventas casi no alcanzarán un aumento del $15 \%$.

0.3 Las ventas tienen bastantes posibilidades de no alcanzar un aumento del $15 \%$.

0.4 Las ventas tienen más posibilidad de no alcanzar un aumento del $15 \%$ que de alcanzarlo.

0.5 Las ventas tienen tantas posibilidades de alcanzar un aumento del $15 \%$ como de no alcanzarlo.

0.6 Las ventas tienen más posibilidad de alcanzar un aumento del $15 \%$ que de no alcanzarlo.

0.7 Las ventas tiene bastantes posibilidades de alcanzar un aumento del $15 \%$.

0.8 Las ventas casi con seguridad alcanzarán un aumento del $15 \%$.

0.9 Las ventas prácticamente alcanzarán un aumento del $15 \%$. 
1 Las ventas alcanzarán un aumento del $15 \%$.

En respuesta a la pregunta planteada, los cuatro expertos contestaron de la siguiente manera:

Experto 1: (0.8)

Experto 2: (0.9)

Experto 3: (0.8)

Experto 4: (0.7)

Se aplica la herramienta de expertizaje a los datos obtenidos, para lo cual se determina la frecuencia normalizada y la frecuencia acumulada como se expresa en la tabla 7 :

TABLA 7.

Expertizaje

\begin{tabular}{llll}
\hline $\begin{array}{l}\text { Escala } \\
\text { endecadaria }\end{array}$ & Vaciado & Normalizar & Acumular \\
\hline 0 & 0 & 0 & 1 \\
0,1 & 0 & 0 & 1 \\
0,2 & 0 & 0 & 1 \\
0,3 & 0 & 0 & 1 \\
0,4 & 0 & 0 & 1 \\
0,5 & 0 & 0 & 1 \\
0,6 & 0 & 0 & 1 \\
0,7 & 1 & 0,25 & 1 \\
0,8 & 2 & 0,5 & 0,75 \\
0,9 & 1 & 0,25 & 0,25 \\
1 & & 0 & 0 \\
TOTAL & 4 & 1 & 8 \\
\hline
\end{tabular}

Entrevistas

Expertizaje $=0.80$

Luego del dato obtenido se debe aplicar el contraexpertizaje, con la aplicación de la siguiente fórmula:

Donde se considera la banda inferior y superior $(\$ 47.058 .776$ - \$56.689.222) de la pregunta sobre al cálculo de las ventas para el próximo año. $\mathrm{Al}$ aplicar la formula se obtiene:

TABLA 8.

Contraexpertizaje

\begin{tabular}{lll}
\hline & 1 & 56689222,3 \\
Contraexpertizaje $=47058776,1+9630446,244$ & 1 & 56689222,3 \\
& 1 & 56689222,3 \\
1 & 56689222,3 \\
& 1 & 56689222,3 \\
& 1 & 56689222,3 \\
& 0,75 & 5668222,3 \\
& 0,25 & 49466387,7 \\
& 0 & 47058776,1 \\
& & 547631331 \\
\hline
\end{tabular}

\section{Entrevistas \\ Contraexpertizaje $=547631331$}

Con el resultado obtenido en la tabla 8 , quedan despejadas las incógnitas del cálculo de las cifras mediante el contraexpertizaje, valor que en última instancia permite disminuir drásticamente la incertidumbre. 
La herramienta de expertizaje y contraexpertizaje es aplicable a cualquier partida contable, lo cual permite obtener valores más cercanos a la realidad y así tomar mejores decisiones en el futuro con la aplicación del dato contable real, enriqueciéndolo con la opinión del experto.

Todas las demás preguntas fueron analizadas de la misma forma que se presentó anteriormente, con el fin de establecer los estados financieros previsionales para que la alta gerencia pueda tomar decisiones.

\subsection{Estados financieros previsionales}

Luego del desarrollo del expertizaje y contraexpertizaje a cada una de las preguntas del presente estudio se arman los balances previsionales. Estos cálculos se realizan partiendo del dato real más la opinión de expertos quienes ayudaron a obtener los siguientes resultados para mejorar las decisiones en el futuro.

TABLA 9.

Estado de Resultados

\begin{tabular}{|c|c|c|c|}
\hline & & & \\
\hline $\begin{array}{l}\text { Estado de } \\
\text { resultados }\end{array}$ & 2017 & & $\begin{array}{l}\text { Proyección } \\
\text { tradicional } \\
2018\end{array}$ \\
\hline $\begin{array}{l}\text { tal } \\
\text { gresos }\end{array}$ & 47622397,64 & 933200,76 & 56859290,01 \\
\hline ostos & 34384648,41 & 39454286,56 & 40721696,09 \\
\hline & 13237749,23 & 1578914,20 & 16137593,92 \\
\hline Gastos & 10161957,41 & 1120 & 11972763,76 \\
\hline Utilio & 3 & $n, 1$ & 4164830,16 \\
\hline dores & 461368,77 & 641361,47 & 624724,52 \\
\hline Imon & 77 & 799563,97 & 885026,41 \\
\hline Jtilidad Neta & 1841839,02 & 2834817,70 & 2655079,23 \\
\hline
\end{tabular}


TABLA 10.

Estado de Situación Financiera

\begin{tabular}{|c|c|c|c|}
\hline \multirow[b]{2}{*}{$\begin{array}{l}\text { Estado de } \\
\text { situación } \\
\text { financiera }\end{array}$} & \multicolumn{3}{|c|}{ Italimentos Cia. Ltda. } \\
\hline & 2017 & $\begin{array}{l}\text { Propuesta } \\
\text { para } 2018\end{array}$ & $\begin{array}{l}\text { Proyección } \\
\text { tradicional } \\
2018\end{array}$ \\
\hline $\begin{array}{l}\text { Activos } \\
\text { Corrientes }\end{array}$ & 13751839,38 & 15702179,63 & 17083257,01 \\
\hline $\begin{array}{l}\text { Activos no } \\
\text { Corrientes }\end{array}$ & 11560471,00 & 16642247,70 & 23520338,47 \\
\hline $\begin{array}{l}\text { Total } \\
\text { Activos } \\
\text { Pasivos }\end{array}$ & 25312310,38 & 32344427,33 & 40603595,48 \\
\hline $\begin{array}{l}\text { Corrientes } \\
\text { Pasivos no }\end{array}$ & 9805068,00 & 10828219,28 & 13205247,96 \\
\hline $\begin{array}{l}\text { Corrientes } \\
\text { Total }\end{array}$ & 3985901,36 & 9001888,34 & 12674628,75 \\
\hline Pasivos & 13790969,36 & 19830107,62 & 25879876,72 \\
\hline $\begin{array}{l}\text { Total } \\
\text { Patrimonio } \\
\text { Total }\end{array}$ & 11521341,02 & 12514319,70 & 14723718,76 \\
\hline $\begin{array}{l}\text { Pasivo + } \\
\text { Patrimonio }\end{array}$ & 25312310,38 & 32344427,33 & 40603595,48 \\
\hline
\end{tabular}

Entrevista y Empresa

Como se evidencia en las tablas anteriores cuando se realiza la proyección de manera tradicional los datos se encuentran sobrevalorados, esto puede darse porque en este método no se toma en consideración la incertidumbre. En cambio, la técnica propuesta arroja datos de mayor aproximación a los reales, se permitirá entonces tomar decisiones más acertadas. Por ello, en el epígrafe siguiente se analizará los indicadores financieros de la propuesta por tener mayor confiabilidad de las cifras.

\section{Resultados}

Luego de haber aplicado la técnica Fuzzy Logic, se procede a realizar el cálculo de los indicadores financieros donde se obtienen los siguientes resultados: 


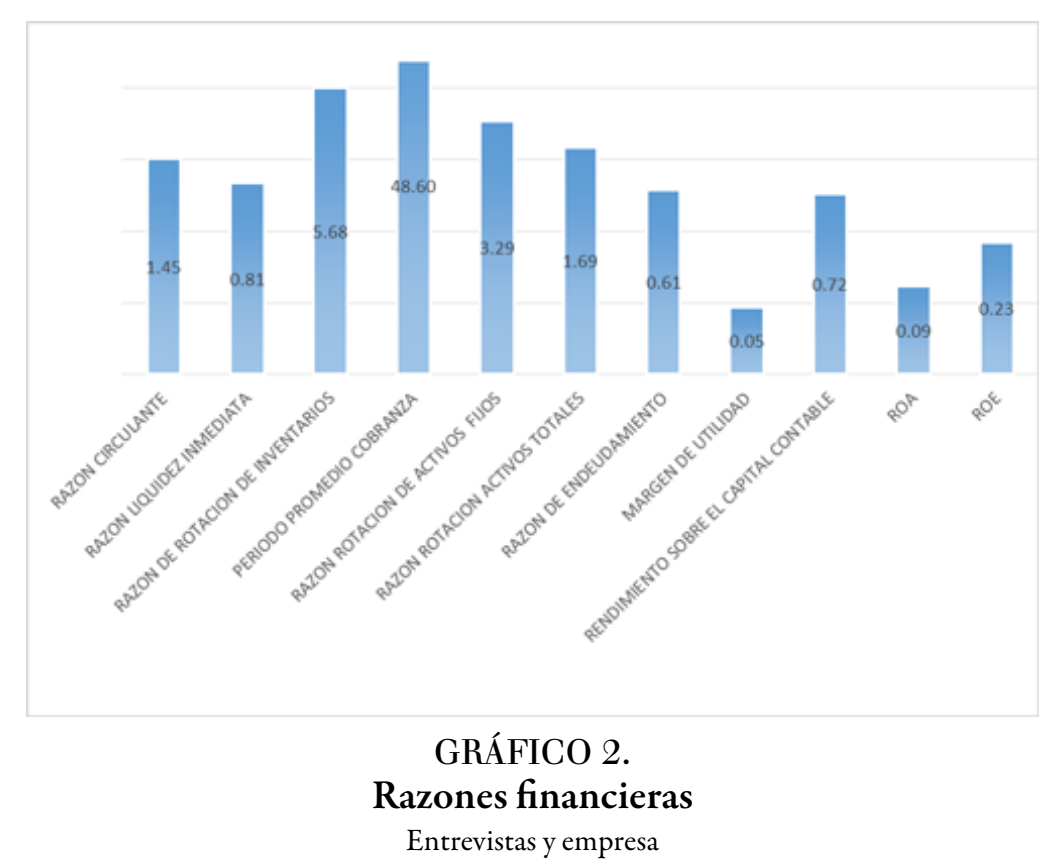

Con la aplicación de la técnica Fuzzy Logic a la empresa Italimentos Cía. Ltda., se construyen los estados financieros previsionales con la opinión de los expertos, dando como resultado lo siguiente:

En el estado de situación financiera, el activo corriente crece en $14 \%$ de un año a otro, el activo no corriente de la empresa crece $43 \%$, mientras que el pasivo corriente aumenta el $14 \%$ y el pasivo no corriente aumenta en $62 \%$.

En cuanto al patrimonio, se refleja un aumento del 8\%, valor que indica como la empresa en el siguiente año tendrá un crecimiento considerable, logrando una buena estructura financiera.

En cuanto al estado de resultados, se observa que la empresa incrementará sus ventas en $16 \%$, sus costos en $14 \%$ y sus gastos en un 10\%, llegando así a tener un aumento en su utilidad, después de impuestos, en 53\%.

Luego de obtener los resultados, se realiza el análisis de ratios financieros, dando al empresario valores que permitan corregir errores reales y vigentes, y así obtener estados con datos más cercanos a la realidad. Al momento de comparar con lo tradicional se obtienen los siguientes resultados:

En cuanto al índice de liquidez en lo tradicional 0.74 y con la propuesta 0.81 , con la razón de endeudamiento en lo tradicional 0.54 y con la propuesta 0.61 , se obtiene de estos indicadores un mayor valor ya que se acerca más a la realidad de la empresa. Con el ROA en lo tradicional se muestra un 0.07 y en la propuesta 0.09 , logrando que la incertidumbre en este índice no se vea afectada. Por su parte, con el ROE en lo tradicional 0.16 y en la propuesta 0.23 , se observa un incremento por ser un dato que se aproxima a la situación real de la empresa.

\section{Conclusiones}

La presente investigación demuestra que mediante la aplicación de la técnica de la lógica difusa, una empresa podrá proveer aspectos que tengan mayor incidencia dentro de la empresa y así, corregir a tiempo anomalías que puedan afectar el buen desempeño de la empresa.

Con el modelo presentado, se parte de una información contable real más la opinión de los expertos, insumo que permite armar los estados previsionales de la empresa para el futuro, es decir, se logra tener tanto el mejor escenario que la empresa puede llegar a percibir, cuanto el peor escenario. Tinto (2015) en su artículo sobre la Automatización Fuzzy aplicado a la contabilidad decisional, concluye al igual que la presente investigación, que para armar los Estados Financieros previsionales, la aplicación de la técnica Fuzzy Logic 
logra automatizar los procesos de expertizaje y contraexpertizaje y así permite atrapar la incertidumbre de los datos obtenidos para los periodos posteriores, situación que permitiría al empresario dinamizar los procesos administrativos y financieros, con el fin de hacer el trabajo de forma eficiente dentro de la empresa, para la toma de decisiones a futuro.

La aplicación del modelo difuso se utilizó para analizar los ratios económicos de la empresa, en donde se encuentra un nivel bajo para el ratio de liquidez, indicador que representa que la empresa está en riesgo de no poder cumplir con sus obligaciones. Por otra parte, el índice de rotación de los activos se encuentra en un nivel estable, lo que indica que la empresa cuenta con inversiones que la hacen sólida y le permitirán crecer en la industria para poder generar mayores ingresos.

En cuanto a los ratios de rentabilidad la empresa genera utilidades y es eficiente para obtener ganancias, maneja de forma adecuada sus gastos y esto le permite determinar si los indicadores que miden a la empresa se encuentran en un estado saludable.

Dado que este método presenta debilidades, se deben tomar los resultados con precaución debido a que se presentan con alto riesgo.

Finalmente, se concluye que el presente método necesita investigaciones en otras áreas para comprobar su bondad en términos de optimizar las decisiones gerenciales.

\section{Referencias bibliográficas}

D’Negri, C. E., \& De Vito, E. L. (2006). Introducción al razonamiento aproximado: lógica difusa. Revista Americana de Medicina Respiratoria, 126-136

Díaz, J., Coba, E., Moreno, K., \& Santamaría, E. (2017). La Lógica Difusa Aplicada a los Ratios Financieros en el Sector Cooperativo del Ecuador. INNOVA , 64-82.

Faga, H. A., \& Ramos Mejía, M. E. (2006). Cómo conocer y manejar sus costos para tomar desiciones rentables. Buenos Aires: Ediciones Granica S.A.

García Parra, M., \& Jordà Lloret, J. M. (2004). Dirección Financiera. Barcelona: Ediciones UPC.

Gil Alvarez, A. (2004). Introducción al Análisis Financiero. Imprenta Gamma, 10.

Lavalle Burguete, A. C. (2016). ANÁLISIS FINANCIERO. Editorial Digital UNID.

Luna, K., Tinto, J., Sarmiento, W., \& Cisneros, D. (2018). Estudio de rentabilidad para el lanzamiento de un nuevo producto aplicando el enfoque difuso. Visión Gerencial, 42-53.

Medina Hurtado, S. (2006). Estado de la cuestión acerca del uso de la lógica difusa en problemas financieros. Cuadernos de Administración, Pontificia Universidad Javeriana, 195-223.

Reig Mullor, J., Sansalvador Selles, M. E., \& Trigueros Pina, J. A. (2000). LÓGICA BORROSA Y SU APLICACIÓN EN LA CONTABILIDAD. REVISTA ESPAÑOLA DE FINANCIACIÓN Y CONTABILIDAD.

Rico F., M. A. (2010). Herramientas con base en subconjuntos borrosos. Propuesta procedimental para aplicar expertizaje y recuperar efectos olvidados en la información contable. Actualidad Contable FACES, 127-146.

Scott, B., \& Brigham, E. F. (2009). Fundamentos de Administración Financiera. México.

Tinto Arandes, J. (2015). Implementación de Tecnología de avanzada (fuzzy-set) aplicadas a los sistemas de control y gestión en las cadenas agroalimentarias del Ecuador. Machala: Universidad Técnica de Machala UTMACH.

Tinto Arandes, J., Molina, M. E., Chavez A., H., \& Mosquera Maldonado, S. (2016). AUTOMATIZACIÓN FUZZY APLICADO EN LA CONTABILIDAD. Revista ECA Sinergia, 7.

Tinto, J., Luna, K., \& Cisneros, D. (2017). Teoría de los efectos olvidados en el rescate de la imagen comercial de los artesanos del calzado en el cantón Gualaceo provincia del Azuay, Ecuador. Visión Gerencial, 24-42.

Tinto, J., Molina, M., \& Chavez, H. (2016). INSTRUMENTOS FUZZY PARA LA TOMA DE DECISIONES EN LAS CIENCIAS CONTABLES. Sinergia, 42-56. 
Tinto, J., Molina, M., Chavez, H., \& Mosquera, S. (2016). AUTOMATIZACIÓN FUZZY APLICADO EN LA CONTABILIDAD. Revista ECA Sinergia, 1-18.

Trillas, E., \& Gutierrez, J. (1992). Aplicaciones de la Logica Borrosa. Madrid: Raycar S.A.

\section{Notas}

1. Alfa cortes, que consiste en trabajar a ciertos niveles de posibilidad de que ocurra el suceso.

2. Análisis en la incertidumbre con base en los números borrosos triangulares en forma de intervalos.

3. Análisis de costos, precio y utilidad bajo el enfoque difuso. La metodología utiliza números borrosos triangulares, que es aquel subconjunto borroso que se forma por una secuencia finita e infinita de intervalos de confianza, que surgen de asignar un nivel de confianza a los valores de un conjunto referencial. Esta aplicación se verá reflejada de la información obtenida de los artesanos de calzado del cantón Gualaceo; los datos hacen relación a los costos totales y la producción realizada en un mes. "La utilización de los números borrosos triangulares y la aplicación de los alfa cortes, permite a los artesanos de calzado, ofertar su producto dentro de una banda de precios, que facilita un mejor manejo financiero para lograr la mejor utilidad posible”. (Tinto, Luna, \& Cisneros, 2017). Generando un aporte cognitivo a los artesanos de calzado considerados en la investigación, con el fin de implementar "este método para la determinación del precio de venta y la utilidad que se desearía obtener entre un numero borroso triangular, con el propósito de que el fabricante puede ofertar su producto dentro de un intervalo, teniendo un precio mínimo y máximo de venta y cuya utilidad está representada de la misma manera" (Tinto, Luna, \& Cisneros, 2017). Los autores Díaz, Coba, Moreno y Santamaría (2017) analizan la Lógica Difusa Aplicada a los Ratios Financieros en el Sector Cooperativo del Ecuador, en donde: Al hablar de lógica difusa se inicia explicando las variables denominadas lingüísticas que permiten analizar perspectivas numéricas de cero $(0)$ a uno (1). Estas variables lingüísticas permiten al experto establecer una valoración cualitativa de datos numéricos que pueden medir el comportamiento difuso de cualquier área de conocimiento (p. 65) En el mismo estudio, se menciona que "al generar el análisis financiero tradicional a través de ratios puede omitirse información relevante al momento de tomar una decisión. En este sentido la lógica difusa puede constituirse en una herramienta que permita dar una mejor perspectiva y para realizar un análisis más profundo de la situación financiera de una organización" (Díaz, Coba, Moreno, \& Santamaría, 2017). Por último, Díaz, Coba, Moreno y Santamaría (2017), explican que la metodología difusa suele utilizarse, de manera general, en casos que presentan complejidad muy alta en el proceso de análisis y por esta situación, es improbable precisar con modelos matemáticos tradicionales, dando paso de esta forma, "a conceptos subjetivos para la precisión del análisis, donde, se puede establecer variables que ayudan a la comprensión total y simplificada de un hecho” (p.66).

(C) Universidad de Cuenca 2019

CC BY-NC-SA

\section{INFORMACIÓN ADICIONAL}

Código JEL: : M41 